\title{
EUV continuum of narrow-line Seyfert 1 galaxies
}

\author{
M. Dietrich ${ }^{1}$, D. M. Crenshaw ${ }^{1}$ and S. B. Kraemer ${ }^{2}$ \\ ${ }^{1}$ Georgia State University, Department of Physics \& Astronomy, Atlanta, GA 30303, USA \\ email: dietrich@chara.gsu.edu, crenshaw@chara.gsu.edu \\ ${ }^{2}$ Catholic University of America, NASA/Goddard Space Flight Center, Code 681, Greenbelt, \\ MD 20771, USA email: stiskraemer@yancey.gsfc.nasa.gov
}

\begin{abstract}
We study the NLR emission line spectrum of 12 NLS1s to constrain the EUV - soft X-ray continuum SED using accurate photoionization modeling. We find that the NLR emission line ratios, which we measure for several NLS1 galaxies, can be reproduced within $\pm 15 \%$ without a strong BBB component in the framework of a two-component model.
\end{abstract}

\section{Introduction}

A common feature in the ionizing EUV continuum of active galactic nuclei is the big blue bump (BBB), generally associated with thermal accretion disk emission (Rees 1984). Strength and shape of the BBB likely depends strongly on black hole mass and accretion rate (Matt et al.1993). The presence of the BBB is indicated by upturns in the far-UV and soft X-rays which are often associated with the high energy tail of the BBB. Recently, Wang \& Netzer (2003) suggested that the soft X-ray properties of NLS1s are natural consequences of their super-Eddington accretion rates. However, Galactic absorption prevents direct access to the EUV range, but it can be mapped measuring the strength of a variety of forbidden optical emission lines that respond to different EUV continuum regions (Netzer et al. 1992; Kraemer \& Crenshaw 2000; Dietrich et al. 2002). Narrow-Line Seyfert 1 galaxies (NLS1s) are excellently suited to investigate the BBB since they show in general strong soft X-ray emission while classical Seyfert 1 galaxies may show only a small or no BBB (Laor et al. 1997; Leighly 2000).

\section{Results and Discussion}

We have observed a sample of 12 nearby, bright NLS1s $\left(m_{V} \leqslant 16^{m}\right)$ using the CTIO $1.5 \mathrm{~m}$ telescope as part of the Small and Moderate Aperture Research Telescope System (SMARTS) observing time. The entire optical wavelength range is covered continuously for $\lambda \lambda \sim 3400-6700 \AA(R \simeq 1000$ to 2000$)$ and with higher resolution for the $\mathrm{H} \beta-$ [OIII] 4959,5007 region $(R \simeq 2000)$.

In contrast to luminous quasars the host galaxy contribution for Seyferts, especially in the optical, can be of the order of $\sim 50 \%$. We analyzed the NLS1 spectra applying a multicomponent fit approach (host galaxy spectrum, power-law continuum, FeII emission, Balmer continuum emission) to isolate the emission line spectrum. To measure the flux of NLR emission lines we used the strong emission line profiles of [OIII] $\lambda \lambda 4959,5007$ as templates to separate the BLR and NLR emission line contributions (Fig. 1).

In contrast to modeling the BLR emission spectrum, a detailed study of the NLR emission avoids the complications of radiative transfer that affect BLR photoionization models. Alexander et al. (1999) and Kraemer et al. $(1999,2000)$ have shown that 

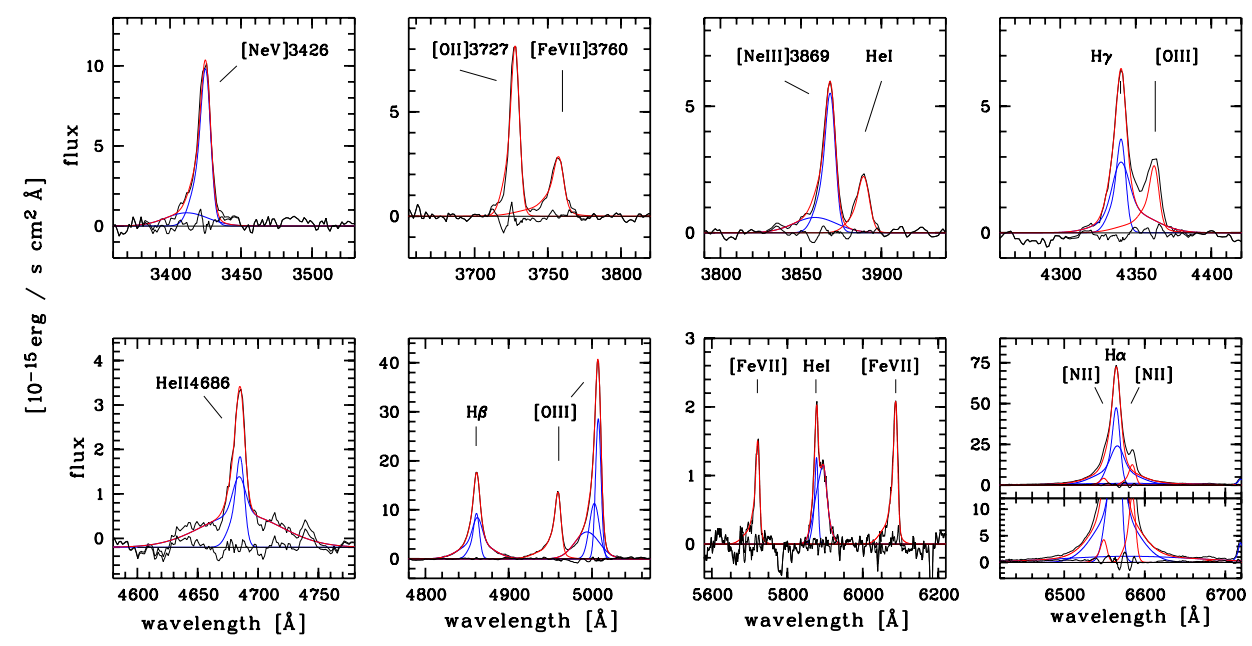

Figure 1. Examples of emission line profile fits of NLR lines for Mkn 1239

accurate photoionization modeling of the NLR emission provide reliable constraints on the ionizing continuum SED. The forbidden high ionization lines like $[\mathrm{NeV}] \lambda 3426$, [NeIII] $\lambda \lambda 3869,3968$, and HeII $\lambda 4686$ are prominent features of the NLR spectrum of the studied NLS1s, indicating a high ionization degree of the gas consistent which earlier studies (e.g., Schmitt 1998).

To reproduce the observed NLR emission line ratios we begin with a single component model. To achieve a better agreement with the observed line ratios we include additional components as necessary. For example, to describe the observed NLR emission line ratios for CTS J13.12 we assume a two component model with solar abundances: (i) high density component (matter bounded): $n_{H}=10^{6.5} \mathrm{~cm}^{-3}, N_{H}=2.5 \times 10^{21} \mathrm{~cm}^{-2}, \mathrm{U}=0.07$ and (ii) low density component (ionization bounded): $n_{H}=10^{3.75} \mathrm{~cm}^{-3}, N_{H}=2.5 \times 10^{21} \mathrm{~cm}^{-2}$, $\mathrm{U}=0.001$. Each component contribute $50 \%$ to the total NLR emission line flux. The ionizing continuum SED is given as a broken power law ( $\alpha=1.5$ up to $1 \mathrm{keV}$ and for higher energies $\left.\alpha=0.8, F_{\nu} \propto \nu^{-\alpha}\right)$. We find that the NLR emission line ratios which we measure for the NLS1 galaxy CTS J13.12, can be reproduced within $\pm 15 \%$ without a strong BBB component in the frame work of a two-component model.

\section{References}

Alexander, T., et al. 1999, ApJ, 512, 204

Dietrich, M., et al. 2002, ApJ, 581, 912

Kraemer, S. B. \& Crenshaw, D. M. 2000, ApJ, 532, 256

Kraemer, S. B., et al. 1999, ApJ, 519, 69

Kraemer, S. B., et al. 2000, ApJ, 531, 278

Laor, A., et al. 1997, ApJ, 489, 656

Leighly, K. M. 2000, New Astronomy Reviews, 44, 395

Matt, G., Fabian, A. C., \& Ross, R. R. 1993, MNRAS, 264, 839

Netzer, H., Laor, A., \& Gondhalekar, P. M. 1992, MNRAS, 254, 15

Rees, M. 1984, ARA\&A, 22, 471

Schmitt, H. R. 1998, ApJ, 506, 647

Wang, J.-M., \& Netzer, H. 2003, A\&A, 398, 927 